\title{
Suitability of Date Palm Leaflets for Sulphated Cellulose Nanocrystals Synthesis
}

\author{
Sherif S. Z. Hindi* \\ Department of Arid Land Agriculture, Faculty of Meteorology, Environment and Arid Land Agriculture, King Abdullaziz University, \\ P.O. Box 80208, Jeddah 21589, Saudi Arabia \\ *Corresponding author: shindi@kau.edu.sa
}

\begin{abstract}
Cellulose nanocrystals (SCNCs) were synthesized from macerated fibers isolated from leaflets of date palm (Phoenix dactylifera L.). The resultant SCNCs were characterized by optical, scanning and transmission electron microscopy, X-ray diffraction (XRD), Fourier transform infrared (FTIR) and thermogravimetric analysis (TGA). $\mathrm{H}_{2} \mathrm{SO}_{4}$-hydrolysis helped isolation of SCNCs with high crystallinity by removing the amorphous regions of the cellulosic microfibril. The SCNCs in an acidic solution were aggregated to form bigger architectures. The SCNCs exhibited a principle sharp peak around $2 \theta=21.25^{\circ}$ related to the cellulose-I structure. The crystallinity index of the SCNCs was found to be high (85.5\%). The average crystallite size of the SCNCs was $2.7 \mathrm{~nm}$. The FTIR results confirmed high purity of the SCNCs conforming to cellulose I. The TGA showed that about $59.13 \%$ of the SCNCs mass was lost up to $500^{\circ} \mathrm{C}$. Based on the results, the leaflets are suitable precursor for SCNCs synthesis.
\end{abstract}

Keywords: acid hydrolysis, cellulose nanocrystals, FTIR, maceration, SEM, TGA, XRD

Cite This Article: Sherif S. Z. Hindi, "Suitability of Date Palm Leaflets for Sulphated Cellulose Nanocrystals Synthesis.” Nanoscience and Nanotechnology Research, vol. 4, no. 1 (2017): 7-16. doi: 10.12691/nnr-4-1-2.

\section{Introduction}

Cellulose is the most abundant biomass material in nature. It can be extracted from natural fibers in different amounts and crystalline properties. The hierarchical and multi-level organization of cellulose allows different kinds of nanoscaled cellulosic fillers [1]. Cellulose also occurs in an amorphous state that is randomly oriented in a spaghetti-like arrangement leading to a lower density compared to nanocrystalline regions [2,3]. On the other hand, the amorphous regions are susceptible to strong acid attack and, under controlled conditions; they may be removed leaving crystalline regions intact [4].

The equatorial positions of the glucopyranose residues stabilize the structure of cellulose, increasing its rigidity and resulting in extensive intra and intermolecular hydrogen bonding that also causes insolubility in water [5]. Removing water from CNF suspensions to maintain nanoscale dimensions of the nanofibrils is a delicate process. [6]. Hydrogen bonds and Van der Waals forces are expected to hold the fibrils together after the removal of water $[7,8]$. There are four methods can be performed to dry the CNCs, namely freeze drying, spray-drying, oven drying and supercritical drying [9].

Many researchers have succeeded to isolate SCNC's by using $\mathrm{H}_{2} \mathrm{SO}_{4}, \mathrm{HCl}$, and $\mathrm{HBr}$. With a sulfuric acid concentration of $63.5 \%(\mathrm{w} / \mathrm{w})$, it was possible to obtain CNCs/whiskers with a length between 200 and $400 \mathrm{~nm}$ and a width less than $10 \mathrm{~nm}$ in approximately $2 \mathrm{~h}$ with a yield of $30 \%$ of initial weight [10]. Beck-Candanedo et al. [11] explained that too long reaction times completely digest the cellulose to yield its component sugar molecules, while lower reaction times will only yield large undispersable fibers and aggregates. The effect of the reaction conditions on CNCs surface charge and sulfur content was not significant and was controlled by factors other than hydrolysis conditions. It was found that sulfuric acid provides more stable aqueous suspensions of cellulosic nanocrystals (CNCs) than hydrochloric acid [12]. This is due to $\mathrm{HCl}$ produces CNCs with minimum surface charge, while $\mathrm{H}_{2} \mathrm{SO}_{4}$ presents a negatively charged surface [4], due to the esterification of surface hydroxyl groups to give charged sulfate groups [11].

Geometrical characteristics such as size, dimensions and shape of CNCs depend on the nature of the cellulose source as well as the hydrolysis conditions such as time, temperature, ultrasound treatment, and purity of materials $[4,11,13]$. Above a critical concentration, the rod-like shape of the charged CNCs leads to the formation of an anisotropic liquid crystalline phase $[11,14]$. Nevertheless, typical dimensions of whiskers range from 5 to $10 \mathrm{~nm}$ in diameter and from 100 to $500 \mathrm{~nm}$ in length. Specific values are provided later.

\section{Materials and Methods}

The study was performed during 2015-2016 in the Central Laboratory at the Agricultural Research Station (ARS), Hada Al-Sham, King Abdul-Aziz University, about $120 \mathrm{~km}$ Northeastern of Jeddah.

\section{Raw Material}

Leaflets of date palm (Phoenix dactylifera L., Sweeda genotype) were chosen for cellulosic fibers production 
(Figure 1a-c) and subsequent SCNCs synthesis. Four healthy trees were selected from those grown at the ARS in a sandy loam soil. The ages of the selected trees were about 20 years old. Four selected fronds were selected according to the four cardinal directions from each tree. After discarding the rachis, the collected leaflets were longitudinally cross cut into chips (about $2 \mathrm{~cm}$ long) using a knife press, chopped, air dried (Figure 1d) and macerated for cellulosic fiber production.

\section{Maceration of cellulosic fibers}

Fifty grams of leaflets chips from each frond were digested separately by using Franklin method (Figure 1e). The digestion solution is composed of both hydrogen peroxide (35\%) and glacial acetic acid in a ratio of 1: 1 and kept, with a compressed cotton stopper, in an oven at $60^{\circ} \mathrm{C}$ for $48 \mathrm{~h}$ or until getting separated fibers with the white aspect [15]. The macerated fibers were removed, disintegrated, washed, air-dried (Figure 1f), characterized and used for SCNC's synthesis.
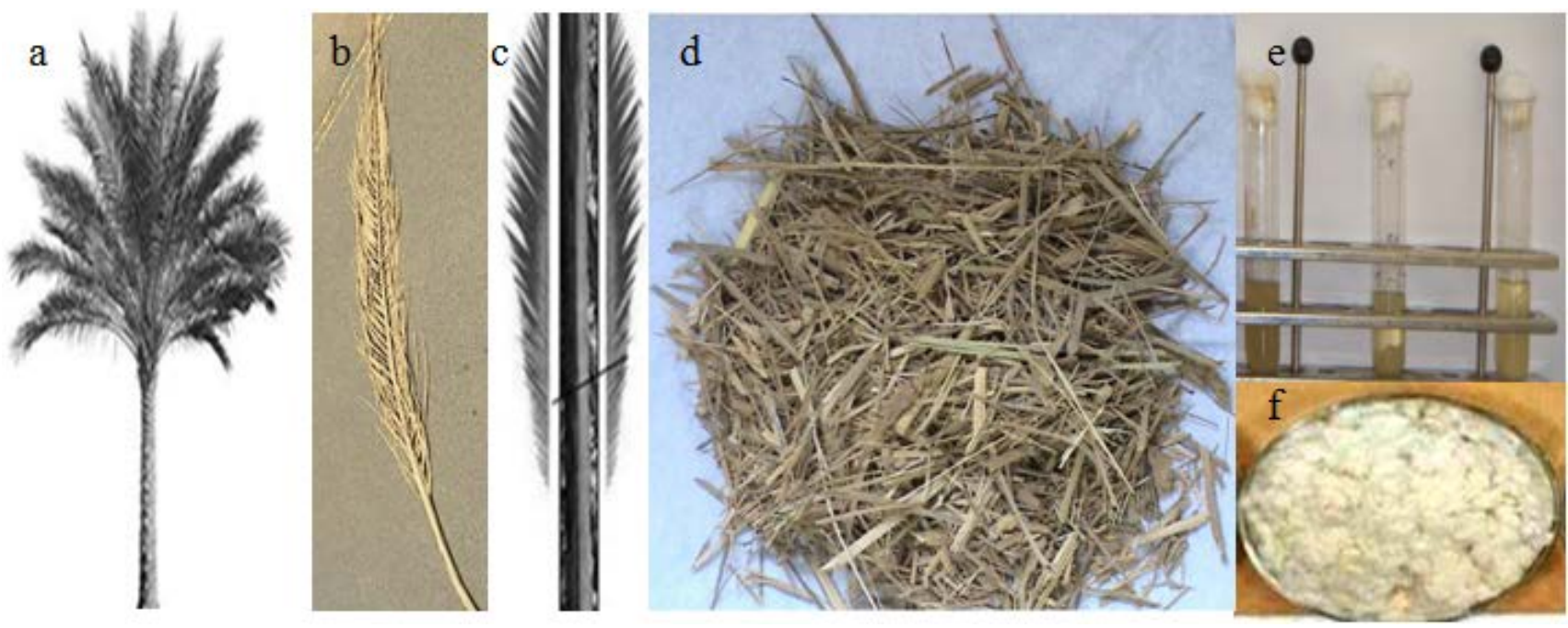

Figure 1. The raw material for production of cellulosic fibers and subsequent cellulose nanocrystals (CNCs) synthesis: a) A date palm tree, b) An airdried frond, c) Isolation of leaflets, d) Chopped air-dried leaflets, e) Maceration process by Franklin method, f) The oven-dried macerated cellulosic fibers
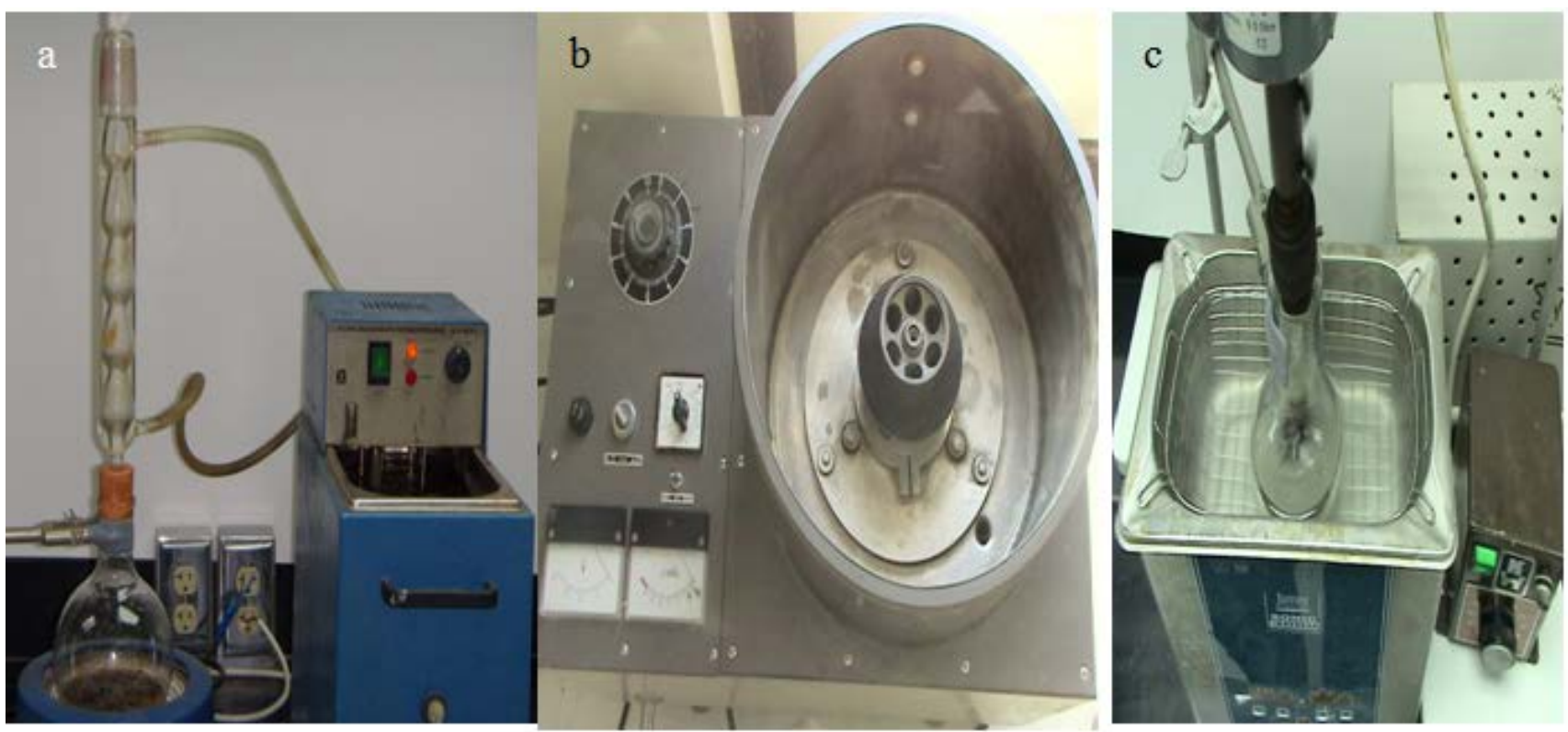

Figure 2. Synthesis of cellulose nanocrystals (CNCs) from macerated fiber of of Phoenix dactylifera leaflet: a) Hydrolysis process, b) Sonication process, and c) The centrifuge device used for the CNCs isolation

\section{Synthesis of SCNCs}

Due to the unique properties for sulphated CNCs, they were synthesized using $\mathrm{H}_{2} \mathrm{SO}_{4}$-hydrolysis (Figure 2a and Figure 7a). Cellulose nanocrystals (CNCs) suspension was prepared from the macerated fibers by sulphuric acid hydrolysis following the recipe used by Cranston and Gray [16] with some modifications and applied by Frone et al. [17]. Hydrolysis was carried out with $64 \%$ w/w sulphuric acid to dissolve the amorphous regions of cellulosic microfibrils producing a CNC-crystallite grafted by sulphated groups (Figure 7a). The hydrolysis process was done at $70^{\circ} \mathrm{C}$ for 30 min with vigorous stirring. This reaction time was selected to guarantee the reaction efficiency and avoid the crystal degradation. Immediately following the acid hydrolysis, the suspension was diluted 20 fold with deionized water to quench the reaction. The suspension was centrifuged by centrifugal separation apparatus type H-600S (Kokusan Enshinki Co. Ltd, Japan) 
at 1500 by (Figure 2b) to remove unhydrolyzed fibers precipitate and then at $14000 \mathrm{rpm}$ for $20 \mathrm{~min}$ to concentrate the cellulose crystals and to remove the excess of aqueous acid. The resultant precipitate was rinsed, re-centrifuged, and dialyzed against deionized water for 5 days until constant neutral $\mathrm{pH}$ was achieved. The suspension was sonicated repeatedly by Vibracell 75043, $750 W$, Bioblock Scientific (Figure 2c) at $30 \%$ output (while cooling in an ice bath) to create cellulose crystals of colloidal dimensions.

\section{Characterization of Cellulose Nanocrystals}

The properties studied for the SCNCs were crystallinity index (CI) and crystallite size (CS) by XRD, functional groups by Fourier transform infrared (FTIR), mass loss by thermogravimetric analysis (TGA).

\section{Sample Preparation for the Different Properties Determinations}

Since SCNCs samples assigned for XRD, FTIR and TGA must be a fine powder, they were ground in a ball mill to passes through a 100 mesh and be retained on a 120 mesh. On the other hand, for the SCNCs samples specified for SEM and TEM spectroscopic studies, a thin film of the SCNCs solution was required for each characterization. This solution was prepared from SCNCs powder completely dissolved in an absolute ethanol by assistance of sonication for SEM and TEM.

\section{The Optical Vision System}

The optical speculation unit used is consisted of a light microscope (CE- MC200A) in a magnification power of $10 \mathrm{X}$ with suitable vision system (OPTIKA PRO 5 Digital Camera- 4083.12) using a Vision PRO 4 software to pick up and processing images as well as to record different measurements of the cells dimensions in a micrometer scale. In addition, the software was also used to show the light intensity of the images (Figure 100).

\section{Scanning Electron Microscopy (SEM)}

SEM study was used to study the surface morphology and types of anatomical features in the tangential plane samples of leaflet tissue as well as the SCNCs. The samples were placed on the double side carbon tape on Al-stub and dried in air. Before examination, all samples were sputtered with a $15 \mathrm{~nm}$ thick gold layer (JEOL JFC- 1600 Auto Fine Coater) in a vacuum chamber $[18,19]$. The specimens were examined with a SEM Quanta FEG 450, FEI, Amsterdam, Netherland. The microscope was operated at an accelerating voltage ranged from $5-20 \mathrm{kV}$.

\section{Transmission Electron Microscopy (TEM)}

This characterization was done only for the resulted SCNCs to speculate their building block and intermediate organelles formed during the crystal growth. The SCNCs were examined by TEM (JEM-1011 JEOL, Japan). The suspension was sampled by using a capillary pipet and dropped onto the copper grid. After being dried for $3 \mathrm{~min}$ at ambient condition, filter paper was used to remove the excess liquid on the copper grid. Afterwards, the dye liquor of phosphotungstic acid was dropped and dyed for 2 min. The dried sample was prepared for observed. The operated voltage was at $100 \mathrm{kV}$.

\section{The X-Ray Diffraction (XRD)}

The X-ray powder diffraction spectra of the fibers were used to study the crystallinity of the using the XRD-D2 Phaser Bruker (USA). The generator was operated at 30 $\mathrm{KV}$ and $30 \mathrm{~mA}$. The samples were exposed for a period of 3000s using CuKa radiation with a wavelength of 0.15418 $\mathrm{nm}$. The sample crystallinity is defined as the ratio of the amount of crystalline cellulose to the total amount of sample material including crystalline and amorphous parts. All the experiments were performed in the reflection mode at a scan speed of $4^{\circ} / \mathrm{min}$ in steps of $0.05^{\circ}$. All samples were scanned in a $2 \theta=26^{\circ}$ range varying from $4^{\circ}$ to $30^{\circ}$.

\section{The Crystallinity Index}

Individual crystalline peaks were first extracted by a curve-fitting process from the diffraction intensity profiles [20,21,22]. The CI was calculated by dividing the diffractogram area of crystalline cellulose by the total area of the original diffractogram. The area under the curve was estimated by summing of adjacent trapezoids using Excel (Microsoft, USA) as indicated by Hindi [23].

\section{The Crystallite Size}

The crystallite size t (nm) of cellulose I structure was calculated by Scherrer equation with respect to the crystallographic plane, namely 002 as follow: Crystallite size $(\mathrm{t})=\mathrm{K} \lambda / \beta_{1 / 2} \operatorname{Cos} \theta$, where $\mathrm{K}$ is the correction factor and usually taken to be $0.91, \lambda$ is the radiation wavelength, $\theta$ is the diffraction angle, and $\beta_{1 / 2}$ is the corrected angular full width at half maximum (FWHM) in radians [23].

\section{Fourier Transform Infrared (FTIR) Spectroscopy}

The FTIR was used to investigate chemical structure of the SCNCs samples using a Bruker Tensor 37 FTIR spectrophotometer. The samples were oven-dried at $100^{\circ} \mathrm{C}$ for 4-5 h, mixed with $\mathrm{KBr}$ in a ratio of $1: 200(\mathrm{w} / \mathrm{w})$ and pressed under vacuum to form pellets. The FTIR-spectra of the samples were recorded in the transmittance mode in the range of $4000-500 \mathrm{~cm}^{-1}$.

\section{Thermal Analysis}

Thermogravimetric analysis (TGA) of the SCNCs were performed by using a Linseis STA PT1000 analyzer. Heating scans were performed from 30 up to $550{ }^{\circ} \mathrm{C}$ at $20^{\circ} \mathrm{C} / \mathrm{min}$ in a nitrogen atmosphere for the CNCs [24,25].

\section{Statistical Design and Analysis}

Completely randomized design with four replications was applied in this study using the analysis of variance procedure and least significant difference test (LSD) at $\mathrm{P} \leq 0.05$ according to El-Nakhlawy [25].

\section{Objectives}

The aim of this work is to:

- Study the suitability of date palm leaflets for sulphated cellulose nanocrystals (SCNCs) synthesis

- Provide some insights into aggregation of the SCNCs after hydrolysis.

\section{Results and Discussion}

\section{Anatomical features of the crude date palm leaflet}

It can be seen from Figure 3 two types of prosenchyma cells in the leaflet tissue, namely fiber cell and vessel cell. The vessel has a thicker wall, a wider lumens and larger volume compared to those for fiber cells. Both prosenchyma elements were found to occupy most of the transverse section area. The dominance of the prosenchyma cells can't be used as an indicator for the leaflet tissue to be a cellulosic resource. This due to the multi-layered cellulose microfibrils are not a pure matrix but are embedded in hemicelluloses and lignin matrices [27]. The holocelluloses content of leaflets' date palm was 
found to be low (16.32\%), while its lignin, ash and total extractives contents were found to be $36.44,10.8$ and $37.06 \%$, respectively [15]. For this reason, the leaflets must be macerated to isolate them and enhance their cellulosic content. In addition, it is preferable for the crude leaflets to be extracted by organic solvents before maceration to eliminate the organic chemicals that interfere with the maceration reagents used [15].

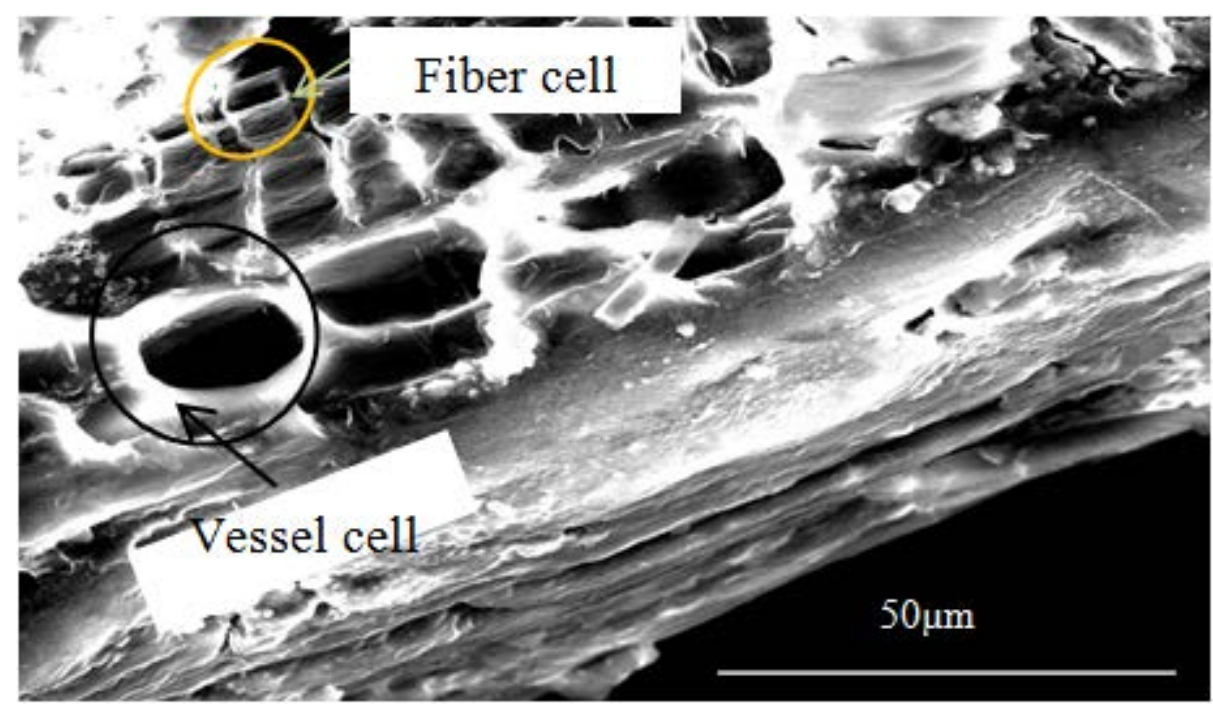

Figure 3. SEM micrograph of two types of prosenchyma cells (Vessel and fiber) in a cross section of crude tissue of Phoenix dactylifera leaflet

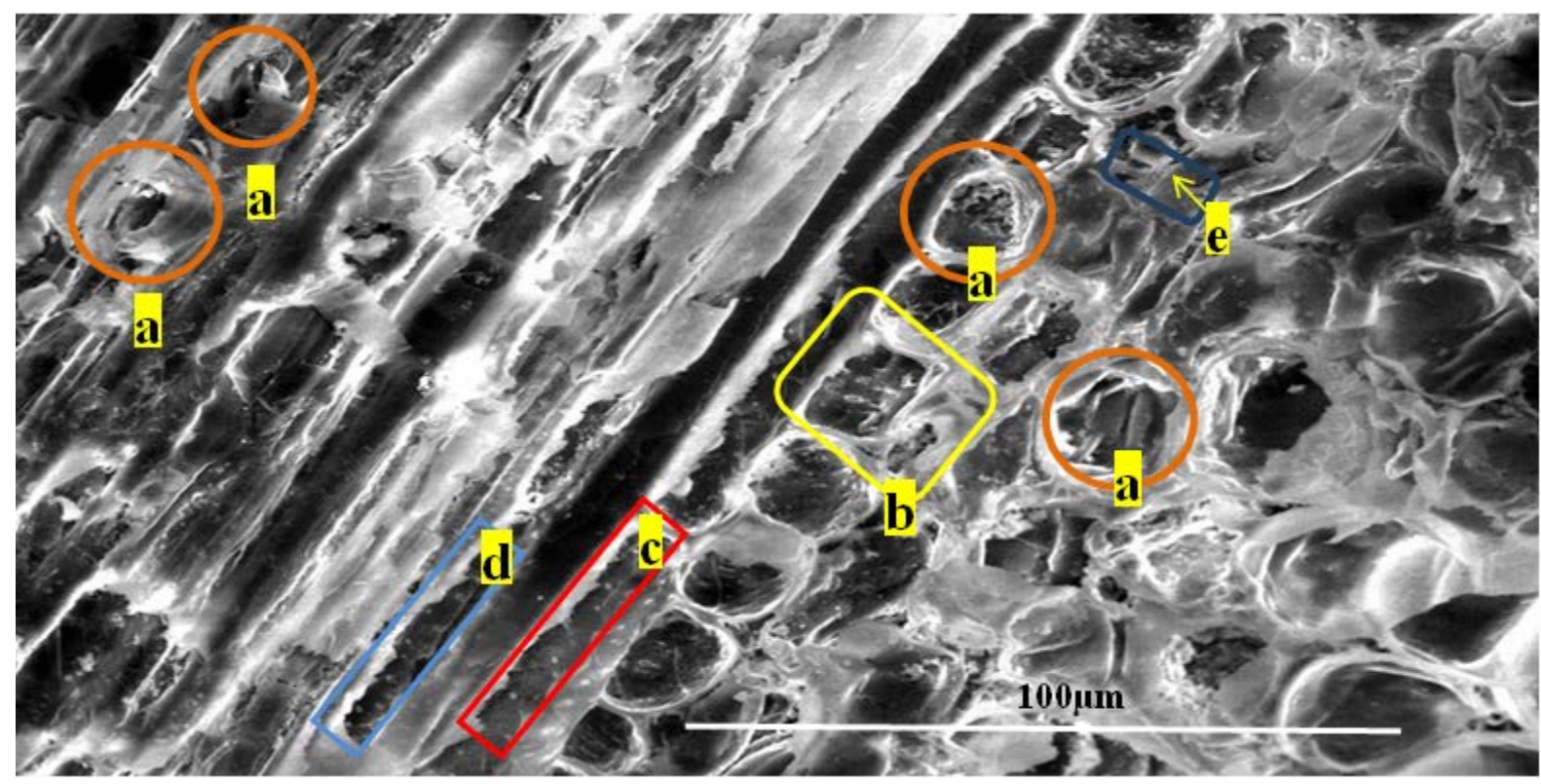

Figure 4. Some anatomical features of crude tissue of Phoenix dactylifera leaflet: a) Stomata, b) Prosenchyma cell (Fiber), c) Parenchyma cell, d) Semiborder pits, and e) border pits

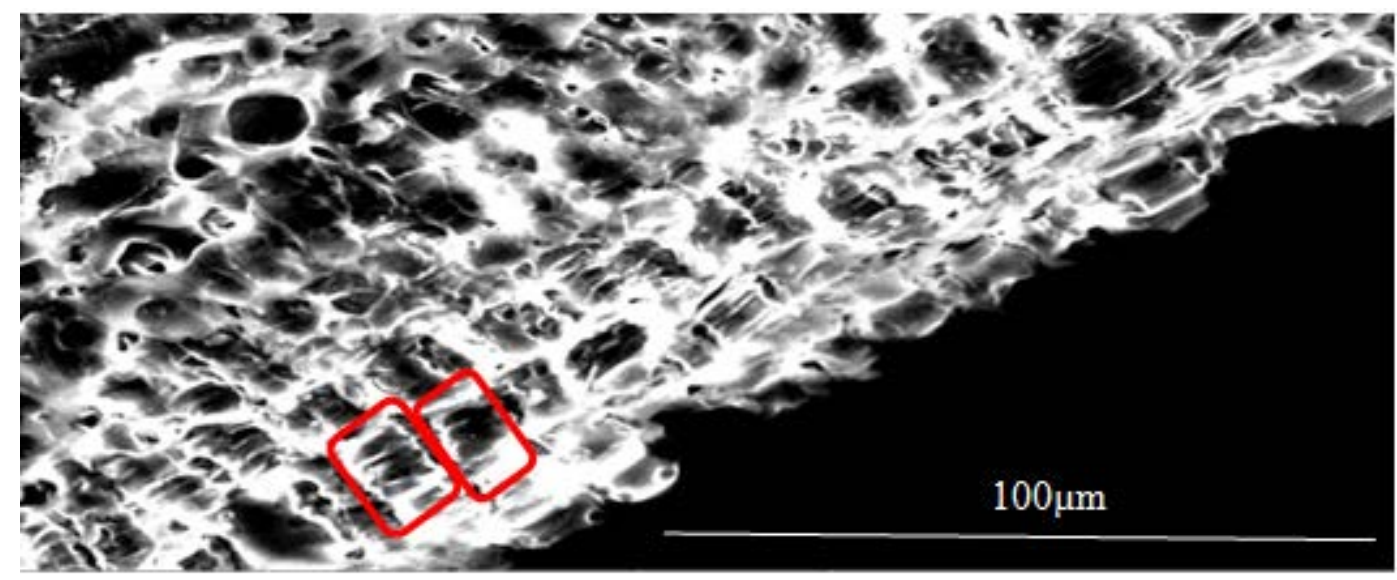

Figure 5. The scalariform-pitting between vessels in a crude tissue of Phoenix dactylifera leaflet 
Other anatomical features of crude tissue of Phoenix dactylifera leaflet, namely stomata, fiber cells parenchyma cell semi-border and border pits are found and presented in Figure 4. Furthermore, the scalariform-pitting used for fluid transition between vessels transversely is clearly shown in Figure 5.

The macerated cellulosic fibers presented in Figure 6 were measured to be $0.733 \mathrm{~mm}$ in length that is lower than that for other date palm genotype $(1.31 \mathrm{~mm})$ found by Hindi et al. [15], while their average width was about $145.36 \mu \mathrm{m}$. These values are comparable to those for hardwood species.

Sulphuric acid hydrolysis helped the isolation of CNCs with high crystallinity index by removing the amorphous regions of the parent microfibrils (Figure 7a). The principle of the disruption of the amorphous regions of cellulose in order to produce CNCs was described by De Souza Lima and Borsali [3]. The hydronium ions can penetrate the cellulosic microfibrils through these amorphous domains promoting the hydrolytic cleavage of the glycosidic bonds releasing individual crystallites [3]. Due to the oxidative action of sulphuric acid along with temperature effect upon hydrolysis, the crystalline domains are exploded into active-charged nano-scaled particles termed as sulphated cellulose nanocrystals (SCNCs). The surfaces of the SCNCs were grafted by sulphated groups (Figure 7a). The SCNCs were arranged in a single colony (Figure 7ba) and/or multiple colonies (Figure $7 \mathrm{bb} \& 7 \mathrm{bc}$ ). It can be observed from Figure 7ba, Figure 7bb, Figure 7bc that the average spherulite diameter was about $5.8 \mathrm{~nm}$ which corresponds to the mean diameter of the cellulosic microfibril width. This means that $\mathrm{H} 2 \mathrm{SO} 4$ cross cut the microfibrils along with their longitudinal axe.

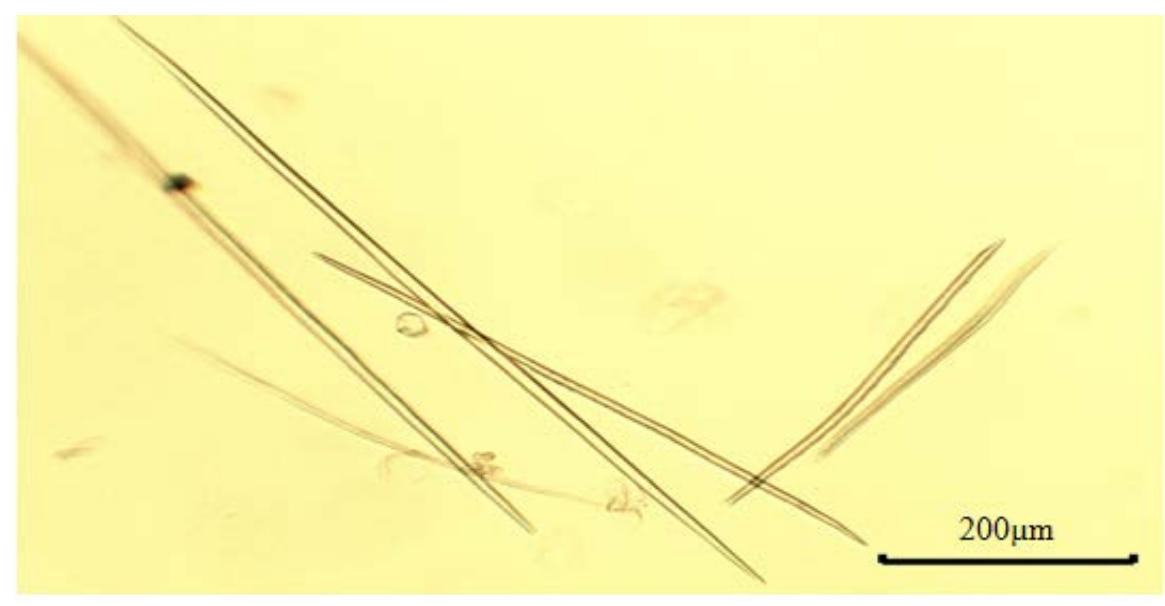

Figure 6. An optical image of macerated fibers of Phoenix dactylifera leaflet

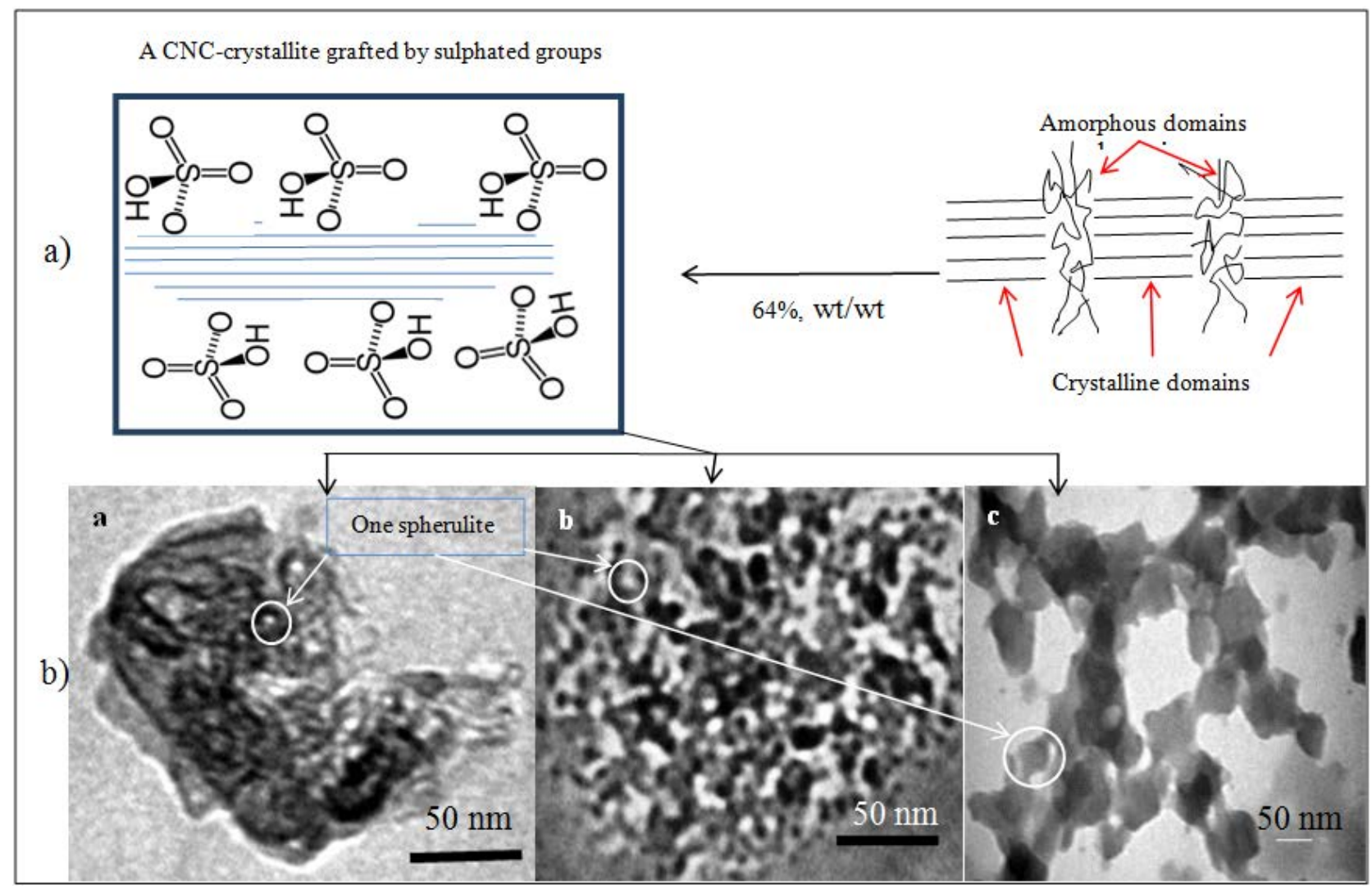

Figure 7. Synthesis of cellulose nanocrystals (SCNCs)” a) A schematic representative of the sulphuric acid-hydrolysis of a part of a cellulosic microfibril, and b) TEM micrographs of active CNCs-spherulites once liberated from their parent microfibrils 

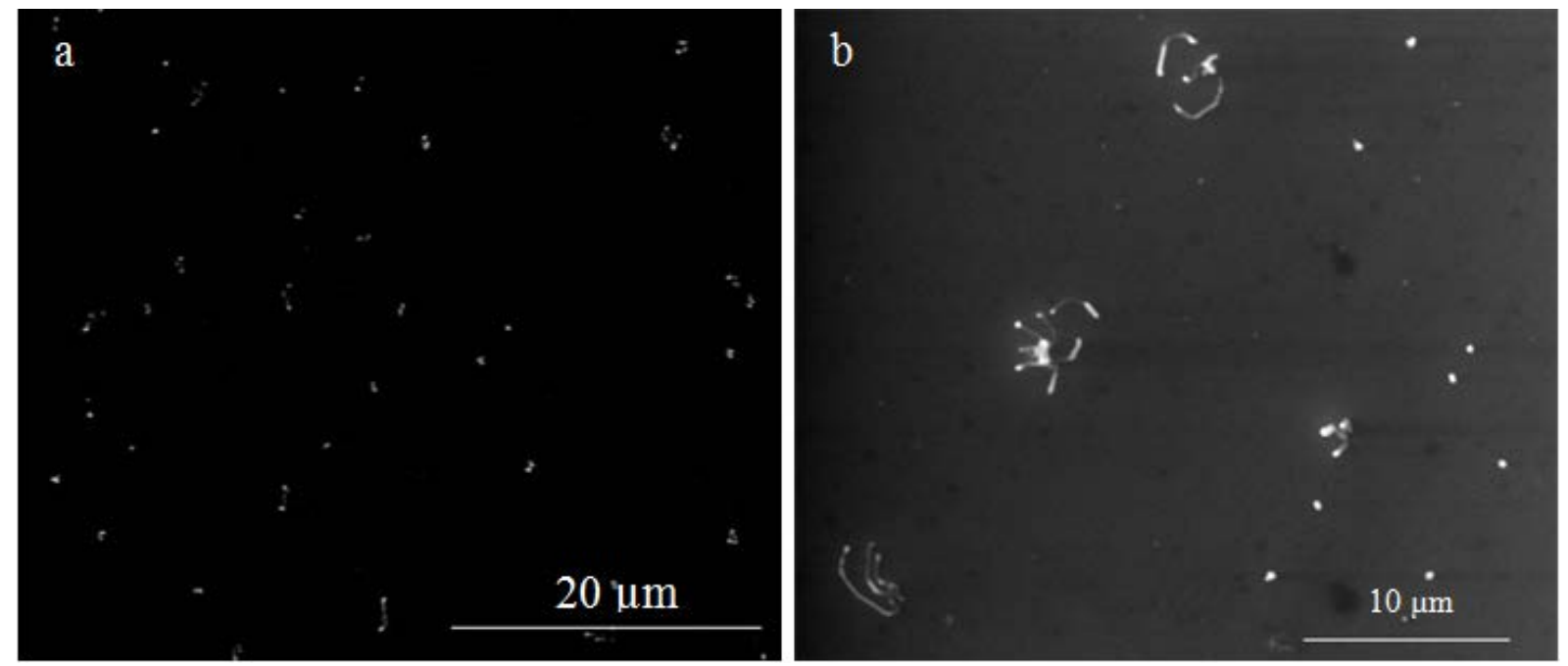

Figure 8. SEM micrograph of SCNCs spherulites: a) converging, and b) aggregating in bigger architectures

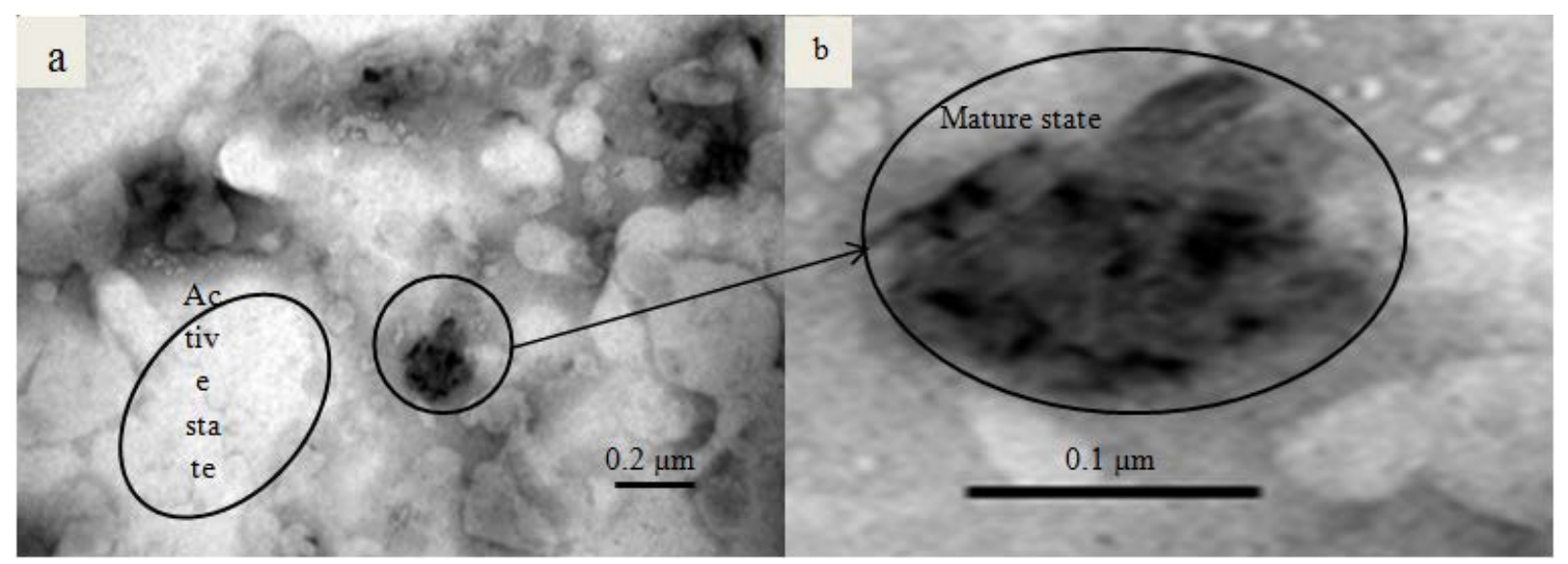

Figure 9. TEM micrographs of SCNCs spherulites aggregated in bigger architectures
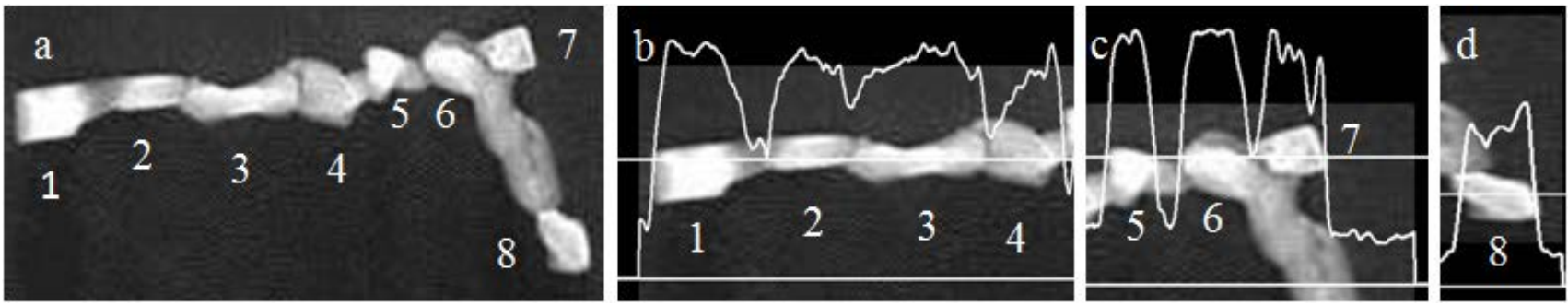

Figure 10. Light intensity of image analysis of the CNC's intermediate construction during crystal growth

Aggregations of crystalline cellulose in micrometric scale are clearly presented in Figure 8a. These particles were more converged and constructed intermediate of final architectures (Figure 8b). These constructions are internally bonded by hydrogen bonds and/or Van der Waals $[8,28]$.

The practical experience in spectroscopy speculation of the SCNCs-supernants upon and after acidic hydrolysis revealed that the recently prepared SCNCs spherulites tend to be glowed in the acidic media (active state). Under the dominant circumstances, they were agglomerated and welded together to form bigger crystalline architectures. After that activity state, the glowing appearance was disappeared in a temporary or permanent state (mature state) with a dark or opaque aspect. These notes can be extracted from Figure 9a and Figure 9b.
It can be seen from Figure 10 that the SCNCs were agglomerated into eight micrometric scale-particles (numbered from 1-8) that were aligned as ready to be welded together (Figure 10a). It is clear that these particles are aglow and brighter than the already welded parts of the SCNCs rods. The brightness of these active sites was confirmed by applying the light intensity analysis. The first four parts (1-4) exhibited four peaks of light intensity (Figure 10b). In addition, the particles no. 5-7 shown in Figure 10c also exhibited three corresponding peaks of the light intensity. Finally, it is clear from Figure 10d that the eighth part of the SCNCs is very bright and shows a clear peak of the light intensity.

For the air- or oven-dried SCNCs, single-, di-, or multispherical agglomerates were arisen with different particle sizes (Figure 11). 


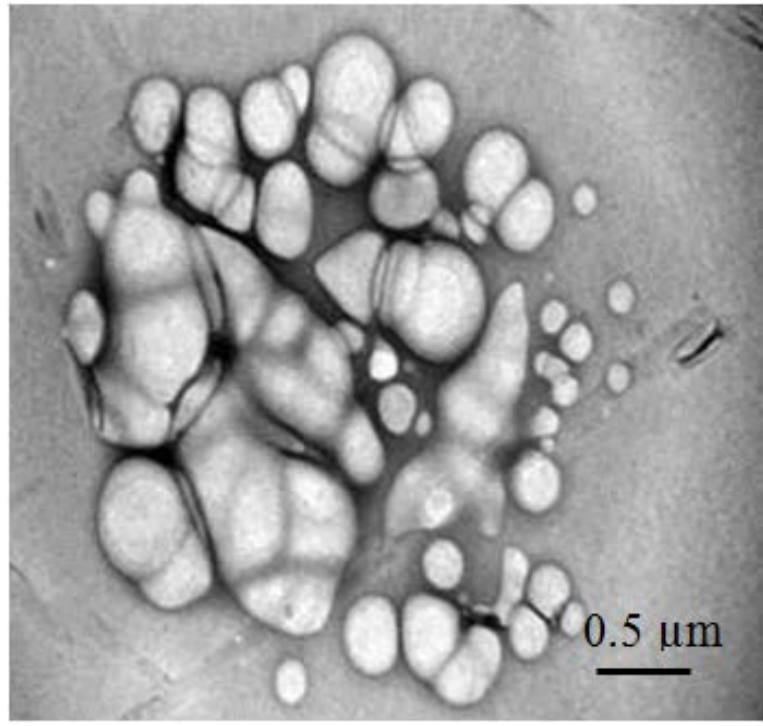

Figure 11. TEM micrograph of oven dried SCNCs: single, di- and multispherulites

\section{X-Ray Diffraction (XRD)}

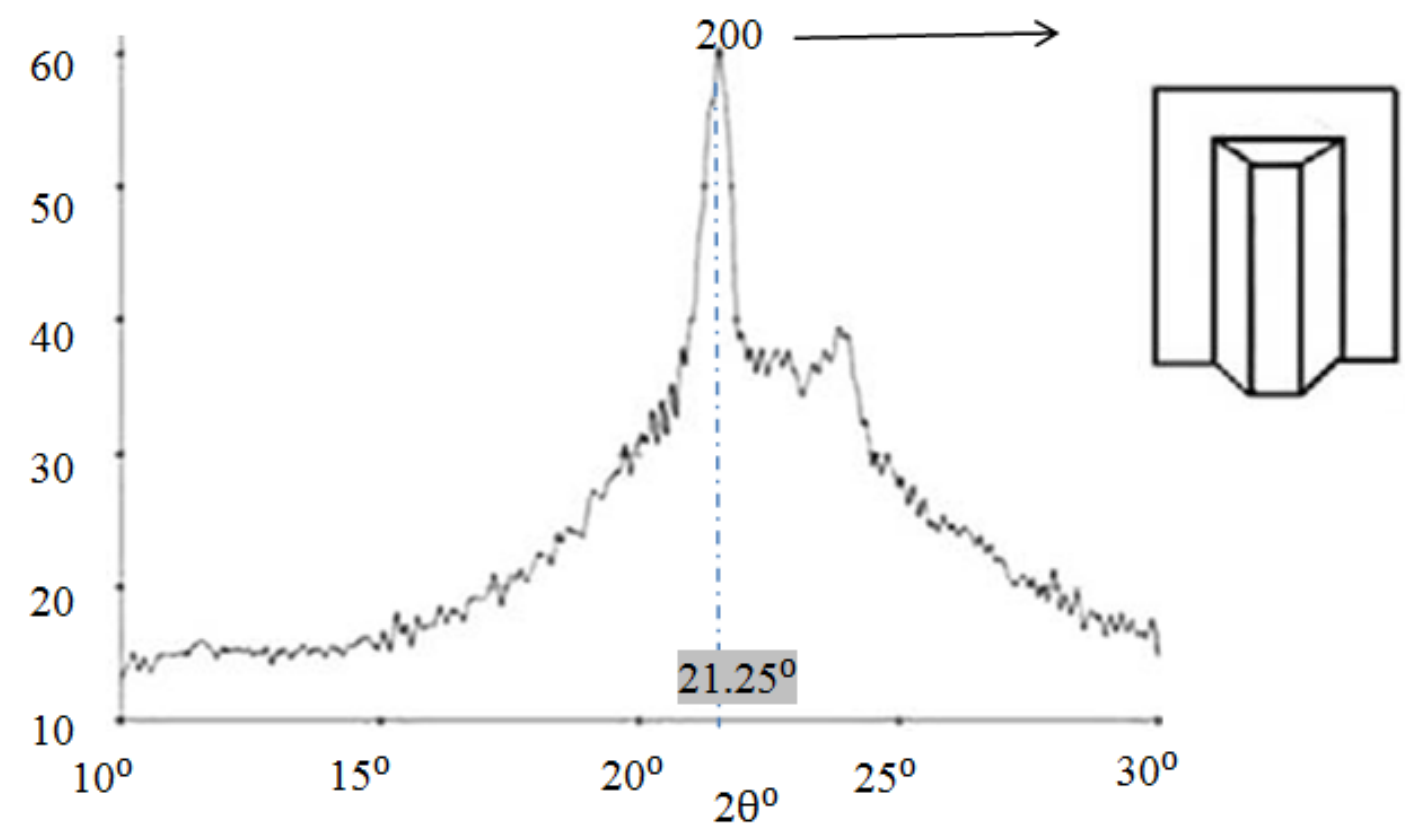

Figure 12. X-ray Diffractogram (XRD) of sulphated cellulose nanocrystals (SCNCs)

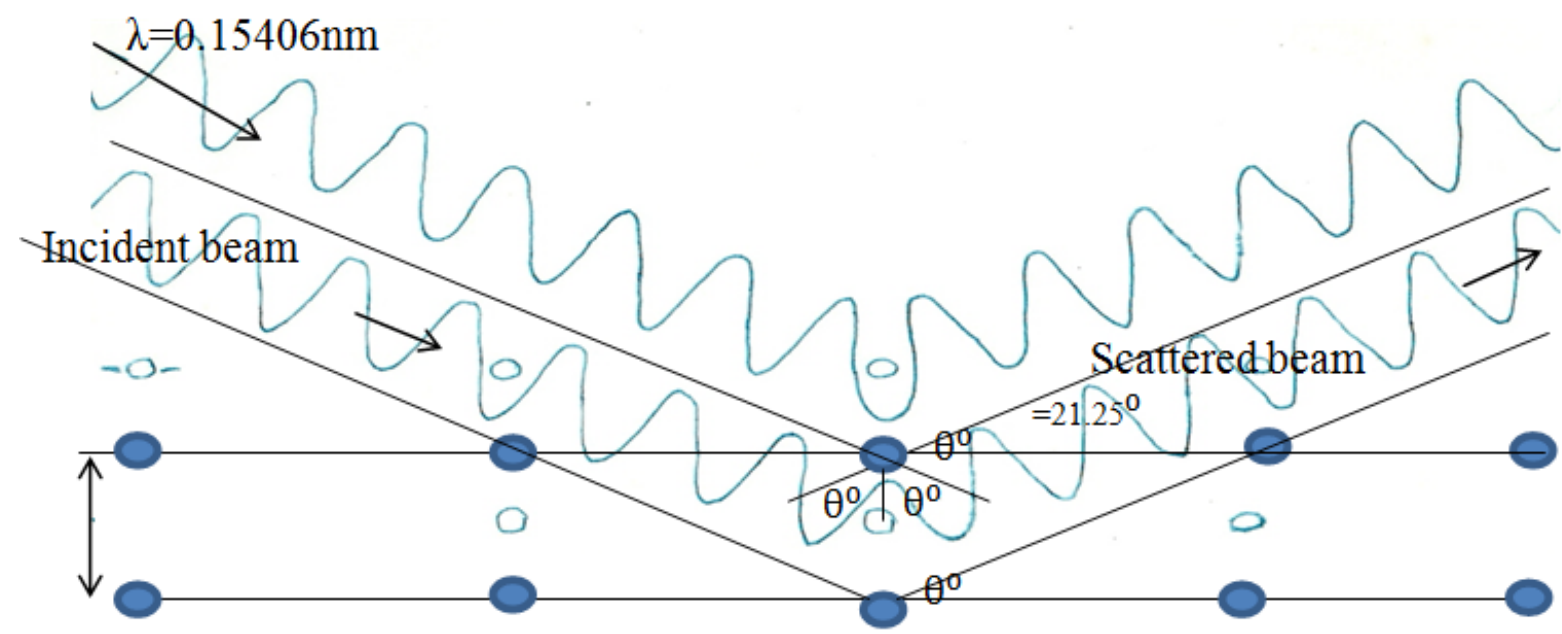

Figure 13. Schematic diagram of Bragg's reflection from lattice planes in the SCNCs 


\section{Fourier Transform Infra-red (FTIR) Spectroscopy}

Changes in chemical functionality after $\mathrm{H}_{2} \mathrm{SO}_{4}$-hydrolysis of the leaflets of date palm were obtained by FTIR spectroscopy as shown in Figure 14. The spectra of the resultant SCNCs show absorption bands of chemical groups characteristic of the crystalline product. All samples presented two main absorbance regions in the range of about $800-1800 \mathrm{~cm}-1$ to $2800-3500 \mathrm{~cm}-1$. The FTIR spectra of all samples have shown sharp bands around the following wavenumbers:

- $1108.46 \mathrm{Cm}^{-1}$ due to $\mathrm{C}-\mathrm{C}$ ring stretching band $\left(\sim 1155 \mathrm{~cm}^{-1)}\right.$ and $\mathrm{C}-\mathrm{O}-\mathrm{C}$ glycosidic ether band $\left(1105 \mathrm{~cm}^{-1}\right)$ [25,33,34,35].

- $1467.21 \mathrm{Cm}^{-1}$ due to scissoring motion of the $\mathrm{CH}_{2}{ }^{-}$ group in the SCNCs [25,33,34,34,35,36].

- $1635.13 \mathrm{Cm}^{-1}$ due to O-H bending of the absorbed water $[25,37,38,39,40,41]$.

- $1737.31 \mathrm{Cm}^{-1}$ due to C-O stretching vibration for the acetyl and ester linkages [25].

- 2852.45-2921.49 $\mathrm{Cm}^{-1}$ due to $\mathrm{C}-\mathrm{H}$ stretching $[25,37,40]$.

- $3438.08 \mathrm{Cm}^{-1}$ due to $\mathrm{O}-\mathrm{H}$ stretching (axial vibration) intramolecular hydrogen bonds for cellulose I [14,42].

Based on the spectral data, it can be confirmed that SCNCs are composed of crystalline cellulose I while content of amorphous cellulose is negligible.

Thermogravimetric analysis (TGA)

The study of thermal properties (TGA) of a material is a dynamic phenomenological approach to investigate the response of sample weight to the changes in temperature. The TGA curves (Figure 15) show changes in SCNCs-weight during heating. The initial mass loss was started at $41.7 \%$ and about $5.88 \%$ of mass was lost up to $100^{\circ} \mathrm{C}$ due to high solvation capacity with water molecule showing evaporation of loose surface bound moisture [25] termed as free water. Furthermore, about $18.89 \%$ of mass was lost upon heating up to $150^{\circ} \mathrm{C}$ due to hygroscopic water known as intermolecularly $\mathrm{H}$-bonded water that was evaporated at near about $120^{\circ} \mathrm{C}$. The thermal degradation of the SCNCs was continued to decrease afterward up to $250^{\circ} \mathrm{C}$, showing a $47.09 \%$ mass loss. In addition, about $59.13 \%$ of the mass was lost up to maximum final temperature of $500^{\circ} \mathrm{C}$.

Thermal degradation of SCNCs occurred at a lower temperature within a broad range of temperature showing lower thermal stability due to their nanometric sizes, greater number of free ends in the SCNCs chains, and may show drastic reduction in the molecular weight and degradation of highly sulfated amorphous regions [33]. This thermal degradation may be corresponded to the degradation of highly sulfated amorphous regions while those degradation occurred at higher temperatures correspond to degradation of unsulfated part of the material [43]. Remnant sulfate groups are responsible for the reduced thermal-stability of the nanocrystals [43] due to the elimination of $\mathrm{H}_{2} \mathrm{SO}_{4}$ in sulfated anhydro-glucose units require less energy [44], therefore, sulfuric acid molecules were released at much lower temperatures during the degradation process.

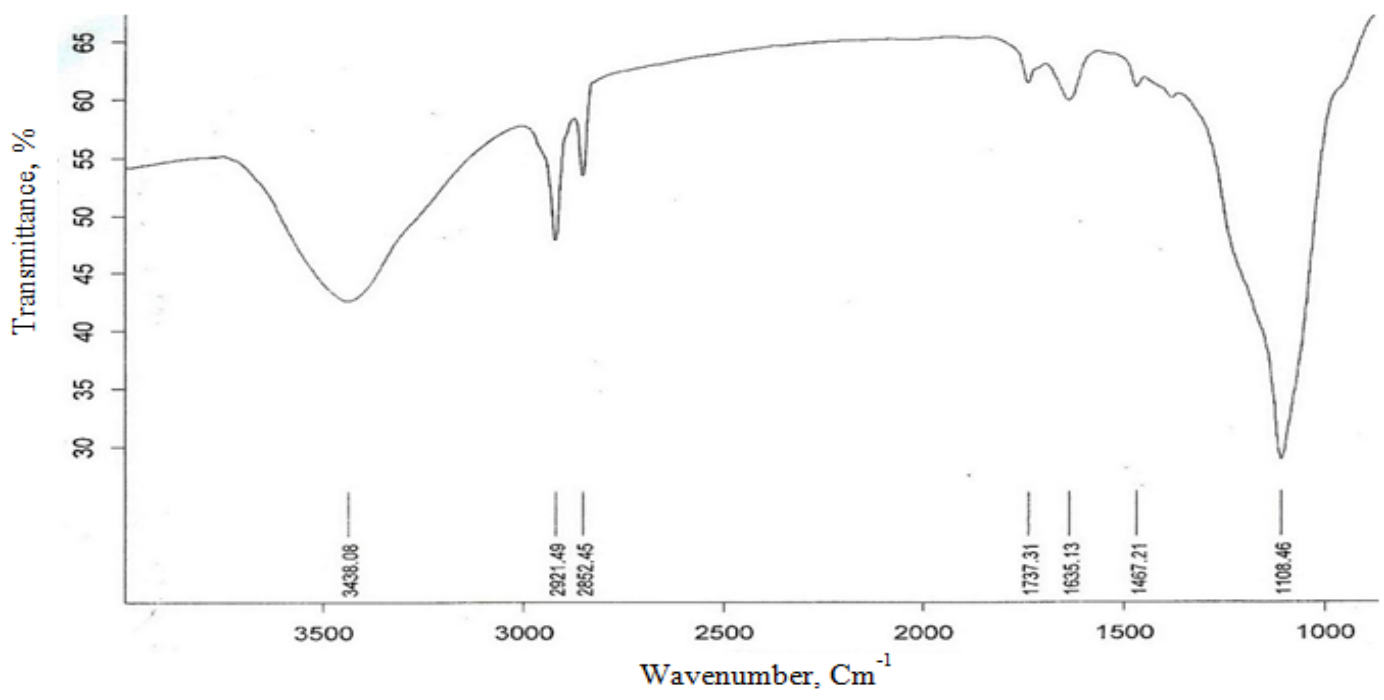

Figure 14. Fourier Transform Infrared (FTIR) Spectroscopy spectra of the cellulose nanocrystals

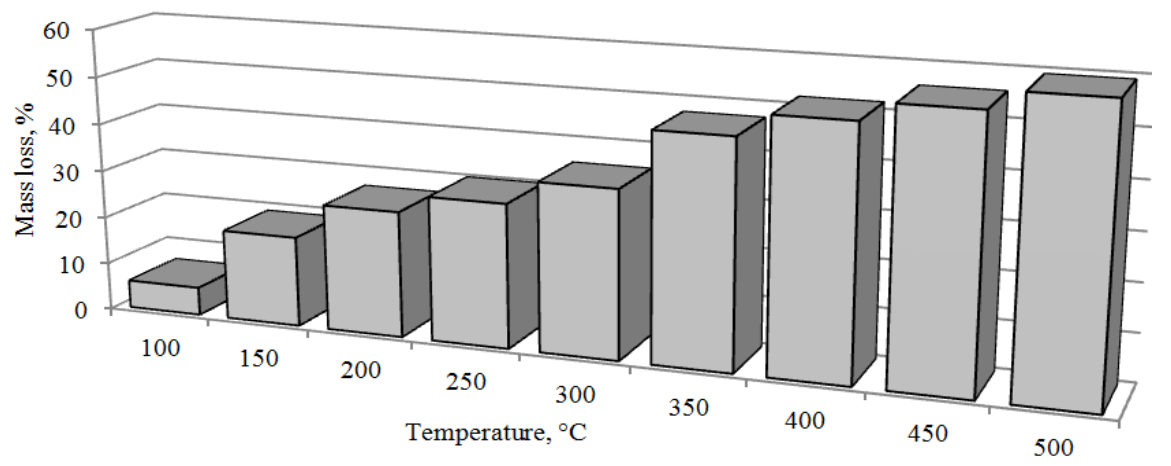

Figure 15. Thermogravimetric analysis (TGA) of the cellulose nanocrystals (CNCs) 


\section{Conclusion}

- Acid hydrolysis helped the isolation of cellulose nanocrystals by removing the amorphous regions of the parent microfibrils.

- The cellulose nanocrystals in the acidic solution were aggregated into different crystalline architectures.

- The cellulose nanocrystals samples exhibited a principle sharp peak around $2 \theta=21.25^{\circ}$ related to hemicelluloses and alpha-cellulose supposing to represent the typical cellulose-I structure.

- The crystallinity index of the cellulose nanocrystals was found to be $85.5 \%$ in which remaining amorphous part was removed during acid-hydrolysis.

- The average crystallite size of the cellulose nanocrystals was $2.7 \mathrm{~nm}$.

- Fourier Transform Infrared spectra of the cellulose nanocrystals confirmed the cellulose nanocrystals are composed of crystalline cellulose I while content of amorphous cellulose is negligible.

- The mass loss of the cellulose nanocrystals was started at 41.7 and about $5.88 \%$ of mass was lost up to $100^{\circ} \mathrm{C}$ due to high solvation capacity with water molecule showing evaporation of free water

- About $59.13 \%$ of the cellulose nanocrystals mass was lost up to maximum final temperature of $500^{\circ} \mathrm{C}$.

- Thermal degradation of the cellulose nanocrystals occurred at a lower temperature within a broad range of temperature showing lower thermal stability.

- Remnant sulfate groups are responsible for the reduced thermal-stability of the cellulose nanocrystals due to the elimination of $\mathrm{H}_{2} \mathrm{SO}_{4}$ in sulfated anhydro-glucose units requires less energy, therefore, sulfuric acid molecules were released at much lower temperatures during the degradation process.

- Based on the results, the leaflets are suitable precursor for SCNCs synthesis.

\section{Acknowledgments}

This project was funded by the Deanship of Scientific Research (DSR), King Abdulaziz University, Jeddah under grant no. 115/155/1432. The author therefore, acknowledges with thanks DSR technical and financial support.

\section{References}

[1] Siqueira, G., Bras, J., and Dufresne, A. 2010. Luffa cylindrica as a lignocellulosic resource of fiber, microfibrillated cellulose, and cellulose nanocrystals. BioResources, 5 (2): 727-740.

[2] Saxena, I. M., Brown, R. M. J. 2005. Cellulose Biosynthesis: Current views and envolving Concepts. Ann. Bot., 96: 9-21.

[3] de Souza Lima, M. M., Borsali, R., 2004. Rodlike Cellulose microcrystals: Structure, properties and applications. Macromol. Rapid Commun., 25: 771-787.

[4] Dufresne, A. 2012. Nanocellulose: From Nature to High Performance Tailored Materials. Walter de Gruyter GmbH \& Co. KG, 475 pp.

[5] Habibi, Y., Lucia, L. A., and Rojas, O. J. 2010. Cellulose Nanocrystals: Chemistry, Self-Assembly, and Applications. Chem. Rev. 2010, 110, 3479-3500.
[6] Peng, Y., Douglas J. Gardner, D. J. and Han, Y. 2012. Drying cellulose nanofibrils: in search of a suitable method. Cellulose. 19 (1): 91-102.

[7] Hiemenz, P. C., and Rajagopalan, R. 1997. Principles of colloid and surface science. CRC Press, New York.

[8] Hunter, R. J. 2001. Foundations of colloid science. Oxford Univ. Press, Oxford.

[9] Pakowski, Z. 2007. Modern methods of drying nanomaterials. Transp Porous Med. 66: 19-27.

[10] Bondeson, D., Mathew, A. and Oksman, K. 2006. Optimization of the isolation of nanocrystals from microcrystalline cellulose by acid hydrolysis. Cellulose. 13: 171-180.

[11] Beck-Candanedo, S., Roman, M., and Gray, D. G. 2005. Effect of reaction conditions on the properties and behavior of wood cellulose nanocrystal. Biomacromolecules.6 (2):1048-54.

[12] Araki, J.; Wada, M.; Kuga, S.; Okano, T. Low properties of microcrystalline cellulose suspension prepared by acid treatement of native cellulose. Colloids Surf. A 1998, 142, 75-82.

[13] Azizi Samir, M.A.S., Alloin, F. and Dufresne, A. 2005. Review of recent research into cellulosic whiskers, their properties and their application in nanocomposite field. Biomacromolecules. 6: 612-626.

[14] Dong, X. M., Revol, J.F., Gray, D. 1998. Effect of microcrystallite preparation conditions on the formation of colloid crystals of cellulose. Cellulose. 5: 19-32.

[15] Hindi S. S. Z., A. A. Bakhashwain and A. A. El-Feel. 2011. Physico-chemical characterization of some Saudi lignocellulosic natural resources and their suitability for fiber production. JKAU; Met. Env. Arid Land Agric. Sci. 21(2): 45-55.

[16] Cranston, E. D. and Gray, D. G. 2008. Birefringence in SpinCoated Films Containing Cellulose Nanocrystals” Colloids Surf. Physicochem. Eng. Aspects. 325: 44-51.

[17] Frone, A. N., Panaitrscu, D. M., Donescu, D. 2011. Some aspects concerning the isolation of cellulose micro- and nano- fibers. U.P.B. Sci. Bull., Series B. 73: 133-152.

[18] Hindi and R. A. Abohassan. 2015. Cellulose triacetate synthesis from cellulosic wastes by heterogeneous reactions. Bioresources. 10 (3): 5030-5048.

[19] Tang, L. G., Hon, D. N. S., and Zhu, Y. Q. 1997. An investigation in solution acetylation of cellulose by microscopic techniques," Journal of Applied Polymer Science. 64 (10): 1953-1960.

[20] Park, S., Baker, J. O., El-Himmell, M., Parilla, P. A., and Johnson, D. K. 2010. Cellulose crystallinity index: Measurement techniques and their impact on interpreting cellulase performance. Biotechnology for Biofuels. 3. 10.

[21] Garvey, C. J., Parker, I. H., and Simon, G. P. 2005. On the interpretation of X-ray diffraction powder patterns in terms of the nanostructure of cellulose I fibres", Macromolecular Chemistry and Physics. 206 (15): 1568-1575, 2005.

[22] Hult, E. L., Iversen, T., and Sugiyama, J. 2003. Characterization of the supennolecular structure of cellulose in wood pulp fibers. Cellulose. 10 (2): 103-110, 2003.

[23] Hindi, S. S. Z. 2017. Some Crystallographic Properties of Cellulose I as Affected by Cellulosic Resource, Smoothing, and Computation Methods. International Journal of Innovative Research in Science, Engineering and Technology (IJIRSET). 6 (1): 732-752.

[24] Fortunati, E., Puglia, D., Monti, M., Peponi, L., Santulli, C., Kenny, J. M. and Torre, L. 2013. Extraction of Cellulose Nanocrystals from Phormium tenax Fibres. Journal of Polymers and the Environment. 21(2): 319-328.

[25] Kumar, A., Negi, Y. S., Choudhary, V. and Bhardwaj, N. K. 2014. Characterization of Cellulose Nanocrystals Produced by AcidHydrolysis from Sugarcane Bagasse as Agro-Waste. Journal of Materials Physics and Chemistry. 2 (1): 1-8.

[26] El-Nakhlawy, F.S. 2008. Principles of statistics, biostatistical experimental design and analysis”. KAU Pub. Center. KSA.

[27] Hindi, S. S. Z. and R. A. Abohassan. 2016. Cellulosic microfibril and its embedding matrix within plant cell wall. International Journal of Innovative Research in Science, Engineering and Technology. 5 (3): 2727-2734.

[28] Hiemenz, P. C., and Rajagopalan, R. 1997. Principles of colloid and surface science. CRC Press, New York.

[29] Kumar, S., Saha, T., Sharma, S. 2015. Treatment of pulp and paper mill effluents using novel biodegradable polymeric flocculants based on anionic polysaccharides: a new way to treat the waste water. Int Res J Eng Technol. 2 (4): 1-14. 
[30] Chen, W. S., Yu, H. P., Liu, Y. X., Chen, P., Zhang, M. X., and Hai, Y. F. 2011. Individualization of cellulose nanofibres from wood using high-intensity ultrasonication combined with chemical pretreatments. Carbohydr. Polym. 83: 1804-1811.

[31] Wada, M., Heux, L., and Sugiyama, J. 2004. Polymorphism of cellulose I family: Reinvestigation of cellulose IV. Biomacromolecules. 5: 1385-1391.

[32] Ciupina, V., Zamfirescu, S., and Prodan, G. 2007. Evaluation of mean diameter values using Scherrer equation applied to electron diffraction images, In: Nanotechnology-Toxicological Issues and Environmental Safety, NATO Science for Peace and Security Series, 231-237.

[33] Mandal, A., and Chakrabarty, D. 2011. Isolation of nanocellulose from waste sugarcane bagasse (SCB) and its characterization”, Carbohydr. Polym. 86: 1291-1299.

[34] Garside, P., and Wyeth, P. 2003. Identification of cellulosic fibres by FTIR spectroscopy: Thread and single fibre analysis by attenuated total reflectance”, Stud. Conser. 48: 269-275.

[35] Nelson, M. L., and O’Connor, R. T. 1964 ${ }^{\mathrm{a}}$. Relation of certain infrared bands to cellulose crystallinity and crystal lattice type. Part I. Spectra of lattice types I, II, III and amorphous cellulose”, J. Appl. Polym. Sci., 8, 1311-1324.

[36] Nelson, M. L., and O'Connor, R. T. 1964 . Relation of certain infrared bands to cellulose crystallinity and crystal lattice type. Part II. A new infrared ratio for estimation of crystallinity in cellulose I and II, J. Appl. Polym. Sci., 8, 1325-1341.
[37] Khalil, H., Ismail, H., Rozman, H. and Ahmad, M. 2001. The effect of acetylation on interfacial shear strength between plant fibres and various matrices. Eur. Polym 37, 1037-1045.

[38] Moran, J.I., Alvarez, V.A., Cyras, V.P., and Vazquez, A., 2008. Extraction of cellulose and preparation of nanocellulose from sisal fibers, Cellulose. 15: 149-159.

[39] Troedec, M., Sedan, D., Peyratout, C., Bonnet, J., Smith, A., Guinebretiere, R., Gloaguen, V., and Krausz, P. 2008. Influence of various chemical treatments on the composition and structure of hemp fibers”, Composites Part A-Appl. Sci. Manufact. 39: 514-522.

[40] Zain, N. F. M., Yusop, S. M. and Ishak Ahmad, I. 2014 Preparation and characterization of cellulose and nanocellulose from pomelo (Citrus grandis) albedo. Nutr Food Sci. 5: 1.

[41] Costa, L. A. de S., Fonseca, A. F., Pereira, F. V. and Druzian, J. I. 2015. Extraction and characterization of cellulose nanocrystals from corn stover. Cellulose Chem. Technol. 49 (2): 127-133.

[42] Li, J. et al. 2014. Homogeneous isolation of nanocellulose by controlling the shearing force and pressure in microenvironment. Carbohyd. Polym. 113: 388-399.

[43] Maren, R., and William, T. W. 2004. Effect of sulfate groups from sulfuric acid hydrolysis on the thermal degradation behavior of bacterial cellulose. Biomacromolecule., 5: 1671-1677.

[44] Julien, S., Chornet, E., and Overand, R. P.1993. Influence of acid pretreatment $\left(\mathrm{H}_{2} \mathrm{SO}_{4}, \mathrm{HCl}, \mathrm{HNO}_{3}\right)$ on reaction selectivity in the vacuum pyrolysis of cellulose. J. Analytic. Appl. Pyrol. 27: 25-43. 